\title{
Clinical Outcomes and Genetic Expression Profile in Human Liver Graft Dysfunction During Ischemia/Reperfusion Injury
}

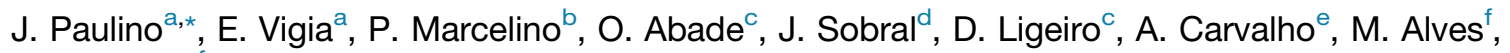 \\ A.L. Papoila ${ }^{f}, H$. Trindade ${ }^{c}$, and E. Barroso ${ }^{a}$ \\ ${ }^{a}$ Centro Hepatobiliopancreático e de Transplantação, Hospital Curry Cabral, ${ }^{b} \mathrm{CEDOC}$, Faculdade de Ciências Médicas, ${ }^{\mathrm{C}} \mathrm{Centro}$ de

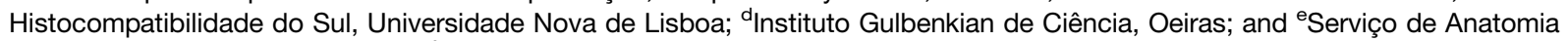 \\ Patológica, Hospital Curry Cabral, 'Departamento de Bioestatística, Universidade Nova de Lisboa, Lisboa, Portugal
}

\begin{abstract}
Introduction. This study aims to compare the molecular gene expression during ischemia reperfusion injury. Several surgical times were considered: in the beginning of the harvesting (T0), at the end of the cold ischemia period (T1), and after reperfusion (T2) and compared with graft dysfunction after liver transplant (OLT).

Methods. We studied 54 patients undergoing OLT. Clinical, laboratory data, and histologic data (Suzuki classification) as well as the Survival Outcomes Following Liver Transplantation (SOFT) score were used and compared with the molecular gene expression of the following genes: Interleukin (IL)-1b, IL-6, tumor necrosis factor- $\alpha$, perforin, E-selectin (SELE), Fas-ligand, granzyme B, heme oxygenase-1, and nitric oxide synthetase.

Results. Fifteen patients presented with graft dysfunction according to SOFT criteria. No relevant data were obtained by comparing the variables graft dysfunction and histologic variables. We observed a statistically significant relation between SELE at T0 $(P=.013)$ and IL-1 $\beta$ at T0 $(P=.028)$ and early graft dysfunction.

Conclusions. We conclude that several genetically determined proinflammatory expressions may play a critical role in the development of graft dysfunction after OLT.
\end{abstract}

$\mathbf{T}$ HE ISCHEMIA/REPERFUSION INJURY (IRI) during liver transplantation (OLT) makes the graft more vulnerable by increasing immunogenicity, and rejection episodes, both earlier and later after OLT [1]. The clinical relevance of these phenomena is related to greater graft dysfunction and loss after OLT [2].

From a hemodynamic standpoint, IRI is well-characterized during OLT surgery, mainly by a decrease in systemic vascular resistance and need for continuous infusion of vasopressors [3-6]. However, the hallmark of IRI is the inflammatory response that lies beyond it.

A serial of molecular events occur after the depletion of cellular adenosine triphosphate during cellular injury and hypoxia. Under normal conditions, this dangerous molecular signaling is directed toward organ preservation and repair; because it is genetically determined, it can vary from patient to patient. During IRI, however, organ damage may be the result of an exacerbated inflammatory reaction. Overproduction of reactive oxygen species occurs, acting during the reperfusion phase [7]. An inflammatory condition is then initiated, by activating Kupffer cells and $\mathrm{T}$ lymphocytes, along with increased production of proinflammatory cytokines (tumor necrosis factor- $\alpha$, interleukin [IL]-1 $\beta$, interferon- $\gamma$, and others), chemokines (macrophage inflammatory protein-2, monocyte chemoattractant protein-1), adhesion molecules, and inflammatory cells recruitment, leading to liver infiltration by neutrophils, and, ultimately, cell death [8].

The authors studied different proinflammatory mediators to characterize the inflammation associated with liver IRI during OLT, as well as their genetic expression, which may result in different inflammatory profiles and different clinical

*Address correspondence to Jorge Paulino, Centro Hepatobiliopancreático e de Transplantação, Hosp. Curry Cabral, Universidade Nova de Lisboa. Hospital Curry Cabral - Rua da Beneficência, 8 - 1069-166 Lisboa, Portugal. E-mail: fusilis@ gmail.com

(c) 2015 by Elsevier Inc. All rights reserved. 360 Park Avenue South, New York, NY 10010-1710 
outcomes, mainly regarding histologic characteristics and post-OLT graft dysfunction.

\section{MATERIALS AND METHODS Patients}

From 2004 to 2011, 856 patients underwent OLT. Data collection was highly dependent on the availability of the surgical team, as well as on the transport of collected samples and laboratory convenience. For this reason, 54 patients were included, not selected by any method, and they expressed the average selection of recipients during the considered period of time. The ages of both recipients and donors were representative of the whole period considered.

We collected patient age, gender, main pathology, blood group, preoperative Model for End-Stage Liver Disease (MELD) score, Child-Pugh score, and Survival Outcomes Following Liver Transplantation (SOFT) score [9]. The highest aminotransferase value after OLT, cold ischemia time, warm ischemia time, and donor age were also collected, as well as the number of red cell packed units and fresh frozen plasma units used during the operation. Most donors were on pressors at the time of harvesting, but there were no detailed data on this issue. All donors had a body mass index of $<30 \mathrm{~kg} / \mathrm{m}^{2}$.

\section{Tissue Sample Collection}

Tissue harvesting was performed by experienced surgical teams in the 4 OLT centers in Portugal, and in all the cases the preservation solution was Celsior (SangStat, Lyon, France) and the rapid Starzl technique was used [10]. Needle biopsies were collected from 54 liver grafts at 3 different times: at the beginning of the harvesting (T0), at the end of the cold ischemia period (T1), and postreperfusion, at the end of the operation (T2).

In the case of familial amyloidotic polyneuropathy (FAP) grafts, informed consent of the donor patients and agreement of the ethical commission of the hospital was obtained.

All biopsy fragments were divided in half; 1 sample was processed for molecular marker expression and the other submitted to a comparative study by usual histologic procedure, following our hospital protocol.

\section{Histologic Analysis}

Tissue fragments were collected in vials containing formaldehyde, and processed in classic hematoxylin and eosin cuts. The histologic classification Suzuki classification [11] was used to quantify the lesions. In addition to the parameters integrating this classification (sinusoidal congestion, vacuolization/ballooning, and necrosis), 2 additional parameters were studied in this issue: the grade of steatosis and neutrophil infiltration. For steatosis, 4 grades were considered: none $(0 \%$, 0 points), slight ( $<30 \%, 1$ point), moderate ( $30 \%-60 \%, 2$ points), and intense ( $>60 \%, 3$ points). Both macrovesicular and microvesicular steatosis were evaluated. Macrovesicular steatosis was considered a reversible condition, defined as a single vacuole pushing the nucleus aside; microvesicular steatosis was associated with deficiency of $\beta$-oxidation of the lipids, and defined as fine and multiple droplets dispersed in the cell cytoplasm. Both were considered able to amplify the IRI in a more relevant way, and enough to induce the phenomena of interference in the hepatic microcirculation (lipopeliosis, especially macrosteatosis).

Neutrophils infiltration was graded as scarce $(\leq 5$ cells per highpower field [HPF; 0 points]), 5-30 cells per HPF (1 point), or $>30$ to $<60$ cells per HPF ( 2 points). To the score provided by the
Table 1. General Characteristics of the Studied Population ( $n=54)$

\begin{tabular}{|c|c|}
\hline Parameter & Result \\
\hline Age $(\mathrm{y})$, mean $\pm S D$ & $45 \pm 14$ \\
\hline Male, n (\%) & $42(78)$ \\
\hline \multicolumn{2}{|l|}{ Blood group, n (\%) } \\
\hline$A$ & $28(52)$ \\
\hline$B$ & $3(6)$ \\
\hline$A B$ & $3(6)$ \\
\hline 0 & $20(37)$ \\
\hline MELD (mean \pm SD) & $16 \pm 8(n=36)$ \\
\hline \multicolumn{2}{|l|}{ CTP score, n (\%) } \\
\hline$A$ & $20(55)$ \\
\hline $\mathrm{B}$ & $10(27)$ \\
\hline $\mathrm{C}$ & $6(18)$ \\
\hline \multicolumn{2}{|l|}{ Main disease, n (\%) } \\
\hline FAP & $15(27)$ \\
\hline $\mathrm{HCV}$ & $16(29)$ \\
\hline Alcoholic cirrhosis & $14(25)$ \\
\hline Biliary diseases & $4(7)$ \\
\hline Autoimmune diseases & $2(3)$ \\
\hline Metabolic diseases & $4(7)$ \\
\hline Acute liver failure & $3(5)$ \\
\hline \multicolumn{2}{|l|}{ SOFT, n (\%) } \\
\hline Low risk & $37(69)$ \\
\hline Low-moderate risk & $12(22)$ \\
\hline High-moderate risk & $5(9)$ \\
\hline
\end{tabular}

Abbreviations: CPT, Child-Turcotte-Pugh; FAP, familial amyloid polyneuropathy; HCV, hepatitis C virus; MELD, Model for End-Stage Liver Disease; SOFT, Survival Outcomes Following Liver Transplantation.

Suzuki classification, we added 2 parameters (steatosis and neutrophil infiltration). According to this final score, the patients were divided in 2 groups: scores of $0-5$ or $\geq 6$.

\section{Molecular and Gene Expression}

The tissue fragments were collected in vials containing RNA later solution (Ambion, Austin, TX). In all the samples, total RNA was extracted and converted in cDNA by reverse transcriptase, and gene expression analysis of a panel of proinflammatory genes was obtained: IL-1b, IL-6, tumor necrosis factor- $\alpha$, perforin, E-selectin (SELE), Fas-ligand, granzyme B, heme oxygenase-1, and inducible nitric oxide synthetase (iNOS2A) by quantitative polymerase chain reaction (PCR) ct, using as reference the genetic expression of sample 1 (nonischemic).

Whenever possible, the service "Assays-on-Demand Gene Expression Products," from Applied Biosystems (Foster City, CA), was used, to synthesize the group of "primers - Taqman probes" specific for each gene studied. A group of Taqman probes for the so-called housekeeping genes ( $\beta$-actin) were used for normalization of the genetic quantification. The PCR kinetic analysis was performed in the sequence detection system of Applied Biosystems ABI Prism 7900. The relative analysis of genetic expression was performed as described by Livak et al [16]. Analysis of relative expression data using real time quantitative PCR and the $2 \mathrm{ct}$ method were performed.

\section{Statistical Analysis and Groups for Comparison}

An exploratory analysis was carried out for all variables. Categorical data are presented as frequencies and percentages, and continuous 
Table 2. Results of Genetic Expression for the Different Studied Genes Related to the Variable Organ Dysfunction

\begin{tabular}{|c|c|c|c|}
\hline Parameters & Dysfunction & Med (P25-P75) & $P$ \\
\hline \multirow[t]{2}{*}{ iNOS at TO } & No & $0.14(0.03-0.30)$ & .381 \\
\hline & Yes & $0.21(0.03-0.68)$ & \\
\hline \multirow[t]{2}{*}{ iNOS at $\mathrm{T} 1$} & No & $0.10(0.03-0.19)$ & .512 \\
\hline & Yes & $0.06(0.01-0.57)$ & \\
\hline \multirow[t]{2}{*}{ iNOS at T2 } & No & $0.06(0.04-0.10)$ & .779 \\
\hline & Yes & $0.05(0.03-0.38)$ & \\
\hline \multirow[t]{2}{*}{ SELE at TO } & No & $0.84(0.44-2.98)$ & .013 \\
\hline & Yes & $2.69(1.35-4.18)$ & \\
\hline \multirow[t]{2}{*}{ SELE at $\mathrm{T} 1$} & No & $1.45(0.54-5.10)$ & .310 \\
\hline & Yes & $2.05(0.99-6.02)$ & \\
\hline \multirow[t]{2}{*}{ SELE at T2 } & No & $2.34(1.57-4.69)$ & .724 \\
\hline & Yes & $2.35(1.20-4.25)$ & \\
\hline \multirow[t]{2}{*}{ FASL at TO } & No & $0.97(0.72-1.86)$ & .745 \\
\hline & Yes & $1.00(0.70-2.81)$ & \\
\hline \multirow[t]{2}{*}{ FASL at T1 } & No & $0.70(0.43-1.68)$ & .209 \\
\hline & Yes & $0.45(0.27-1.33)$ & \\
\hline \multirow[t]{2}{*}{ FASL at T2 } & No & $0.22(0.10-0.47)$ & .573 \\
\hline & Yes & $0.29(0.13-0.64)$ & \\
\hline \multirow[t]{2}{*}{ GRZB at TO } & No & $1.42(0.86-2.70)$ & .566 \\
\hline & Yes & $2.96(0.58-4.91)$ & \\
\hline \multirow[t]{2}{*}{ GRZB at $\mathrm{T} 1$} & No & $1.36(0.65-3.78)$ & .900 \\
\hline & Yes & $0.53(0.44-9.76)$ & \\
\hline \multirow[t]{2}{*}{ GRZB at T2 } & No & $1.68(0.96-4.41)$ & .742 \\
\hline & Yes & $2.65(0.78-5.33)$ & \\
\hline \multirow[t]{2}{*}{$\mathrm{HO}-1$ at TO } & No & $266.29(146.74-385.47)$ & .323 \\
\hline & Yes & $289.80(179.39-652.55)$ & \\
\hline \multirow[t]{2}{*}{$\mathrm{HO}-1$ at $\mathrm{T} 1$} & No & 193.79 (147.75-275.31) & .798 \\
\hline & Yes & $202.33(135.82-460.55)$ & \\
\hline \multirow[t]{2}{*}{$\mathrm{HO}-1$ at $\mathrm{T} 2$} & No & $164.32(125.05-353.36)$ & .742 \\
\hline & Yes & $160.16(99.72-270.90)$ & \\
\hline \multirow[t]{2}{*}{ IL-6 at TO } & No & $0.37(0.10-2.50)$ & .354 \\
\hline & Yes & $0.60(0.20-3.54)$ & \\
\hline \multirow[t]{2}{*}{ IL-6 at $\mathrm{T} 1$} & No & $2.26(0.74-4.85)$ & .423 \\
\hline & Yes & $1.62(0.23-4.97)$ & \\
\hline \multirow[t]{2}{*}{ IL-6 at T2 } & No & $6.40(2.38-14.36)$ & .634 \\
\hline & Yes & $7.82(3.31-12.10)$ & \\
\hline \multirow[t]{2}{*}{ IL-1 Beta at T0 } & No & $9.84(2.54-19.39)$ & .028 \\
\hline & Yes & $19.53(12.10-40.88)$ & \\
\hline \multirow[t]{2}{*}{ IL-1 Beta at T1 } & No & 22.67 (8.47-46.82) & .682 \\
\hline & Yes & $25.39(7.87-217.55)$ & \\
\hline \multirow[t]{2}{*}{ IL-1 Beta at T2 } & No & 36.35 (17.96-89.83) & .171 \\
\hline & Yes & 61.38 (53.19-86.65) & \\
\hline
\end{tabular}

variables as mean or median, standard deviation $(\mathrm{SD})$ or interquartile range (25th-75th percentile). Univariable analysis was done using nonparametric tests $\left(c^{2}\right.$ or Fisher exact test, Mann-Whitney and Kruskal-Wallis) because of the existence of outliers, high variability, and skewed distributions.

The significance level $\alpha=0.05$ was considered and $95 \%$ CI were calculated as appropriate. All data were analyzed using the Statistical Package for the Social Sciences for Windows version 19.0 (SPSS Inc, Chicago IL). The main outcome considered was de graft dysfunction according to SOFT criteria, compared to histologic data and data from studied gene's expression. The preservation injury and early graft dysfunction according to Howard and Ploeg-Maring criteria were also evaluated, although not subjected to specific analysis.

\section{RESULTS}

The main characteristics of enrolled patients are presented in Table 1. In the studied cohort, we observed graft preservation injury in 5 cases, early graft injury (according to Ploeg-Maring criteria) in 9 patients, and 15 patients presented graft dysfunction according to SOFT criteria. The comparison between graft dysfunction and clinical variables are presented in Table 2. Although not attaining significance, we observed male patients were 3 times less likely to develop graft dysfunction. For cold ischemia time, graft dysfunction occurred 1.9 more often in patients with a cold ischemia time of $>500$ minutes, and 5 times more often in patients with a warm ischemia time of $>90$ minutes. In 2 of the 16 cases that experienced graft dysfunction, the liver graft came from a living donor with FAP. In only 8 cases of these 16 the donor was $<50$ years old. Among grafts that developed dysfunction, there was a donor-recipient gender mismatch, which affected female recipients (5/6) much more often than male recipients (4/10).

The comparison between graft dysfunction and histologic variables is presented in Table 3. No relevant associations were found. Nonetheless, we observed that, in patients with baloonization at $\mathrm{T} 0$ and $\mathrm{T} 1$, the possibility to develop graft dysfunction was $\leq 7.5$ times higher, and the presence of necrosis at $\mathrm{T} 0$ and $\mathrm{T} 1$ conferred a 2.3 times greater probability to develop graft dysfunction.

Laboratory and gene expression data are presented in Table 4. We observed a significant relationship between SELE at T0 $(P=.013)$ and IL-1 $\beta$ at T0 $(P=.028)$ and early graft dysfunction. Significant associations were confirmed on multivariate analyses, but only regarding SELE expression at T0 (odds ratio [OR], 1.93; 95\% CI, 1.06-3.50; $P=.030$ ).

Concerning the expression of IL- $1 \beta$ at $\mathrm{T} 0$, the comparison between cases of patients with and without dysfunction revealed a $P$ value of .028 (Med [P25-P75], no 9.84 [2.54-19.39]; yes, 19.53 [12.10-40.88], Mann-Whitney).

\section{DISCUSSION}

We have found that patients expressing higher levels of SELE and IL-1 $\beta$ at T0 presented more severe early graft function after OLT, before flushing with the recipient's blood, at T0. This suggests that inflammatory phenomena may influence critically the severity of IRI during OLT. Interestingly, no histologic data correlated with IRI, although baloonization and necrosis were more frequent at $\mathrm{T} 0$, as well as with a cold ischemia time of $>500$ minutes and

Table 3. Multivariate Analysis for Clinical Variables and Demographic Data for Graft Dysfunction

\begin{tabular}{lccc}
\hline \multicolumn{1}{c}{ Variables } & OR & $95 \% \mathrm{Cl}$ & $P$ \\
\hline Male gender & 0.13 & $0.01-0.25$ & .077 \\
Cold ischemia $(\mathrm{min})$ & 1.74 & $0.95-3.18$ & .074 \\
Warm ischemia $(\mathrm{min})$ & 1.64 & $0.91-2.94$ & .099 \\
SELE expression in T0 & 1.93 & $1.06-3.50$ & .030 \\
\hline
\end{tabular}

Abbreviations: OR, odds ratio; SELE, E-selectin. 
Table 4. Univariate Analysis of Gene Expression in All Time Points Based on Graft Dysfunction

\begin{tabular}{|c|c|c|c|c|}
\hline Gene & All Patients $(n=54)$ & Patients Without Graft Dysfunction & Patients With Graft Dysfunction & $P^{*}$ \\
\hline \multicolumn{5}{|c|}{ FASL median $\left(\mathrm{P}_{25}-\mathrm{P}_{75}\right)$} \\
\hline TO & $0.98(0.72-2.16)$ & $0.97(0.72-1.86)$ & $1.00(0.70-2.81)$ & .873 \\
\hline T1 & $0.68(0.34-1.50)$ & $0.70(0.43-1.68)$ & $0.45(0.27-1.33)$ & .776 \\
\hline T2 & $0.25(0.11-0.52)$ & $0.22(0.10-0.47)$ & $0.29(0.13-0.64)$ & .366 \\
\hline \multicolumn{5}{|c|}{ GRB median $\left(\mathrm{P}_{25}-\mathrm{P}_{75}\right)$} \\
\hline TO & $1.51(0.61-3.70)$ & $1.42(0.86-2.70)$ & $2.96(0.58-4.91)$ & .677 \\
\hline T1 & $1.33(0.47-5.72)$ & $1.36(0.65-3.78)$ & $0.53(0.44-9.76)$ & .857 \\
\hline T2 & $2.08(0.94-4.52)$ & $1.68(0.96-4.41)$ & $2.65(0.78-5.33)$ & .282 \\
\hline \multicolumn{5}{|c|}{ HO1 median $\left(\mathrm{P}_{25}-\mathrm{P}_{75}\right)$} \\
\hline TO & 276.60 (153.41-419.45) & $266.29(146.74-385.47)$ & 289.80 (179.39-652.55) & .167 \\
\hline $\mathrm{T} 1$ & $198.06(139.91-291.16)$ & $193.79(147.75-275.31)$ & $202.33(135.82-460.55)$ & .801 \\
\hline T2 & $162.24(120.97-315.76)$ & $164.32(125.05-353.36)$ & 160.16 (99.72-270.90) & .900 \\
\hline \multicolumn{5}{|c|}{ IL-6 median $\left(P_{25}-P_{75}\right)$} \\
\hline T0 & $0.48(0.15-2.64)$ & $0.37(0.10-2.50)$ & $0.60(0.20-3.54)$ & .282 \\
\hline T1 & $1.62(0.50-4.77)$ & $2.26(0.74-4.85)$ & $1.62(0.23-4.97)$ & .571 \\
\hline $\mathrm{T} 2$ & $7.19(2.76-13.42)$ & $6.40(2.38-14.36)$ & $7.82(3.31-12.10)$ & .662 \\
\hline \multicolumn{5}{|c|}{ IL- $1 \mathrm{~b}$ median $\left(\mathrm{P}_{25}-\mathrm{P}_{75}\right)$} \\
\hline TO & $12.17(2.83-24.01)$ & $9.84(2.54-19.39)$ & $19.53(12.10-40.88)$ & .143 \\
\hline T1 & $22.67(8.31-49.51)$ & 22.67 (8.47-46.82) & $25.39(7.87-217.55)$ & .342 \\
\hline T2 & $49.91(22.40-85.84)$ & $36.35(17.96-89.83)$ & $61.38(53.19-86.65)$ & .788 \\
\hline \multicolumn{5}{|c|}{ iNOS2A median $\left(\mathrm{P}_{25}-\mathrm{P}_{75}\right)$} \\
\hline TO & $0.15(0.03-0.32)$ & $0.14(0.03-0.30)$ & $0.21(0.03-0.68)$ & .578 \\
\hline T1 & $0.09(0.02-0.19)$ & $0.10(0.03-0.19)$ & $0.06(0.01-0.57)$ & .791 \\
\hline T2 & $0.06(0.03-0.13)$ & $0.06(0.04-0.10)$ & $0.05(0.03-0.38)$ & .895 \\
\hline \multicolumn{5}{|c|}{ SEAL median $\left(\mathrm{P}_{25}-\mathrm{P}_{75}\right)$} \\
\hline TO & $1.17(0.49-3.26)$ & $0.84(0.44-2.98)$ & $2.69(1.35-4.18)$ & .214 \\
\hline T1 & $1.53(0.61-5.45)$ & $1.45(0.54-5.10)$ & $2.05(0.99-6.02)$ & .517 \\
\hline T2 & $2.30(1.40-4.56)$ & $2.34(1.57-4.69)$ & $2.35(1.20-4.25)$ & .319 \\
\hline
\end{tabular}

${ }^{*} P$ value obtained by comparison of genes between groups of patients with and without graft dysfunction by logistic regression model.

a warm ischemia time of $>90$ minutes, a situation related to donor's condition and harvesting.

Unlike other selectins, SELE is expressed in an inducible manner, not under basal conditions, but in response to inflammatory stimulations, both chronic and acute, by endothelial cells, and not by hepatic sinusoids [12]. Among these stimuli is IL-1 $\beta$, a molecule involved in the precocious phases of the inflammation cascade [13].

Interestingly, the association between these 2 proinflammatory genes and the tendency toward graft dysfunction was only documented at T0, without any influence of recipient factors. It is recognized that any surgical manipulation triggers a molecular reaction through activation of Kupffer cells [14], explaining the enhanced expression of those 2 genes even before reperfusion, as an intrinsic quality associated with the graft itself [15]. The increase occurring at $\mathrm{T} 0$ in association with graft dysfunction represents a clinical implication with therapeutic potential.

In previous study [16] on this cohort, livers harvested from FAP patients presented less neutrophil infiltration at T0 $(P=.001)$. We linked the neutrophil infiltration with gene expression of HO1 in biopsies taken at T2 $(P=.022)$, as well as with the molecular expression of genes related to an attenuated proinflammatory reaction during IRI, namely, iNOS2A at T0 and HO1 at T2. We concluded that livers from FAP donors express differently the genes related to attenuating proinflammatory reactions, and present less neutrophil infiltration during harvesting. These findings add to the previous description of a better short-term outcome in patients receiving FAP liver grafts. These differences prompted us to try to identify these inflammatory responses to organ dysfunction after OLT in the whole cohort.

The inflammatory process during IRI is well known $[17,18]$. Nonetheless, patients can react differently, according to a genetically mediated predisposition in the release of proinflammatory cytokines, which can explain, at least partially, the different and hardly preventable clinical features (mostly hemodynamic) and consequences (graft dysfunction and loss) of IRI. Nonetheless, obtained data point in 2 different, but proximal, directions. SELE is oriented to lymphocyte infiltration and IL- $1 \beta$ to inflammation.

Selectins are members of cell adhesion molecules, whether those we studied are secreted by lymphocytes. They are linked to lymphocyte homing and promote cellular-mediated inflammation and infiltration, leading to acute and chronic inflammatory processes. Because we could not demonstrate these facts with histologic findings, it is hard to prove these basic aspects of SELE as a reference for IRI. Nonetheless, this description may reserve attention in further studies. The most studied process evolving SELE is metastatic tumor spread [19]. Diapedesis of tumor cells from the circulation into secondary sites is believed to occur through a mechanism 
similar to that of leukocyte extraversion, in which there must be contact and then the cells roll along the endothelial cell layer. SELE is an adhesion molecule that is not expressed on normal endothelial cells; however, SELE is expressed transiently on the surface of vascular endothelium after stimulation with IL- 1 and tumor necrosis factor- $\alpha$. Their role in IRI has been described in both cellular infiltration and molecular signaling. Interfering with selectin produces a protective effect against liver IRI [20]. The improvement of hemodynamics and decreased leukocyte adherence after treatment with $\mathrm{N}$-acetylcysteine might result from the shedding of selectins.

The role of IL- $1 \beta$ can more interesting. IL- $1 \beta$ is cleaved by caspase 1 (IL-1 $\beta$ convertase) and is linked to inflammation, cell proliferation and differentiation, and apoptosis. This is an inflammatory process, now recognized as an autoinflammatory process, and observed as a mechanism involved in some autoinflammatory diseases $[21,22]$. Autoinflammatory diseases diverge from autoimmune diseases, mediated by abnormal cell-mediated injury, mainly lymphocytes and macrophages. They are the result of uncontrolled release of inflammatory mediators, as the result of intracellular inflammasomes, a complex of proinflammatory-producing protein complexes, produced when cells sign the "danger" of damage. These phenomena are mediated genetically and can vary from person to person. The caspase 1 complex is a part of this inflammatory pathway and it may be related to IRI phenomena, regardless of the organ involved, including OLT. It originates in large, multiprotein complexes that sense danger signals through specific receptors. These protein complexes are known as inflammasomes, and are determined genetically. Regarding liver inflammatory processes, a number of conditions have been linked to this inflammatory pathway. Inflammosomes regulate cell fate and pyroptosis, which differ from apoptosis by damaging plasma cell membrane and lack of chromatin condensation, leading to the secretion of various danger molecules. The clinical entities involved are drug-induced liver injury, endotoxin-induced liver injury, and alcoholic and nonalcoholic fatty liver diseases [23]. IRI processes, which are linked with liver resection, liver manipulation, hypovolemia, and OLT, have been linked to this proinflammatory pathway as well. The data presented herein support these findings, demonstrating the importance of inflammasomes and IL-1 $\beta$ release in the process of IRI. Other known data, such as cold ischemia time, warm ischemia time, and other specific donor conditions, cannot be ignored and are described widely [24,25]. Nonetheless, the different clinical outcomes of IRI and the different clinical presentation, can be, at least partially, attributed to different gene expression of IL- $1 \beta$ in donor livers, leading to a more significant inflammatory process during IRI in OLT [26,27].

In most cases of dysfunction in this cohort, there was a gender mismatch, which affected female recipients $(5 / 6)$ much more than male recipients $(4 / 10)$. There are in literature very few reports on this issue, and most are related to lesser graft survival, specifically regarding male recipients and female donors [28,29]; however, the arguments presented are related with the hormonal milieu of the donor and its action on the colangiocytes [30].

The only recipient pathology directly implicated with graft survival concerning gender mismatch is chronic hepatic disease with hepatitis $\mathrm{C}$ virus infection [31], wherein a female donor is an independent predictor of fibrotic evolution and graft loss [32]. However, this issue does not apply to this study, because only 1 of the 12 female recipients in group of 54 patients presented with fibrosis. There are some reports on poor prognosis in female recipients with donor mismatch, which is considered an independent risk factor for primary graft nonfunction [33]. The causes are yet to be clarified, but the role of minor histocompatibility antigens such as H-Y antigen, codified by genes from Y chromosome, has been suggested in literature; further studies are awaited [34].

\section{Study Limitations}

The main limitation of this study is the small number of patients enrolled. Many variables can be accounted for, but no previous selection was performed, and patients were enrolled randomly. In this regard, more studies with greater numbers of participants are needed.

We decided not to exclude FAP grafts from deceased donor grafts; it is well-known that FAP grafts experience less damaging phenomena, all previous to harvesting. As we previously described, histologic data differ in these 2 types of graft. Accordingly, proinflammatory data can also differ. It should be also pointed out that gene expression related to histologic findings was different from that described herein. This matter should be elucidated further.

In conclusion, several genetically determined proinflammatory expressions may play a critical role in the development of graft dysfunction after OLT. We found that gene expression of IL-1 $\beta$ and SELE in donor livers were linked with liver dysfunction after OLT.

\section{REFERENCES}

[1] Zhai Y, Petrowsky H, Hong JC, et al. The clinical relevance of these phenomena are related to greater graft dysfunction nad loss after LT. Nat Rev Gastroenterol Hepatol 2013;10:79-89.

[2] Paugam-Burtz C, Kavafyan J, Merckx P, et al. Postreperfusion syndrome during liver transplantation for cirrhosis: outcome and predictors. Liver Transpl 2009;15:522-9.

[3] Aggarwal S, Kang Y, Freeman JA, et al. Postreperfusion syndrome: cardiovascular collapse following hepatic reperfusion during liver transplantation. Transplant Proc 1987;19:54-5.

[4] Toledo-Pereyra LH. Definition of reperfusion injury in transplantation. Transplantation 1987;43:931-2.

[5] Hilmi I, Horton C, Planisic RM, et al. The impact of post reperfusion syndrome on short-term patient and liver allograft outcome in patients undergoing orthotopic liver transplantation. Liver Tanspl 2008;14:504-8.

[6] Nanashima A, Pillay P, Crawford M, et al. Analysis of postrevascularization syndrome after orthotopic liver transplantation: 
the experience of an Australian liver transplant center. J Hepatobiliary Pancreat Surg 2001;8:557-63.

[7] Jaeschke H. Reactive oxygen and mechanisms of inflammatory liver injury: present concepts. J Gastroenterol Hepatol 2011;26(suppl 1):173-9.

[8] Diesen DL, Kuo PC. Nitric oxide and redox regulation in the liver: part II. Redox biology in pathologic hepatocytes and implications for intervention. J Surg Res 2011;167:96-112.

[9] Reichert B, Becker T, Weismuller TJ, et al. Value of the preoperative SOFT-score, P-SOFT-score, SALT-score and labMELD-score for the prediction of short-term patient and graft survival of high-risk liver transplant recipients with a pre-transplant labMELD-score >/=30. Ann Transplant 2012;17:11-7.

[10] Starzl TE, Miller C, Broznick B, et al. An improved technique for multiple organ harvesting. Surg Gynecol Obstet 1987;165:343-8.

[11] Suzuki S, Toledo-Pereyra LH, Rodriguez FJ, et al. Neutrophil infiltration as an important factor in liver ischemia and reperfusion injury. Modulating effects of FK506 and cyclosporine. Transplantation 1993;55:1265-72.

[12] Coito AJ. Leukocyte transmigration across endothelial and extracellular matrix protein barriers in liver ischemia/reperfusion injury. Curr Opin Organ Transplant 2011;16(1):34-40.

[13] Futosi K, Fodor S, Mocsai A. Neutrophil cell surface receptors and their intracellular signal transduction pathways. Int Immunopharmacol 2013;17(3):638-50.

[14] Schemmer P, Enomoto N, Bradford BU, et al. Activated Kupffer cells cause a hypermetabolic state after gentle in situ manipulation of liver in rats. Am J Physiol Gastrointest Liver Physiol 2001;280(6):G1076-82.

[15] Kolaczkowska E, Kubes P. Neutrophil recruitment and function in health and inflammation. Nat Rev Immunol 2013;13(3):159-75.

[16] Livak KJ, Schmittgen TD. Analysis of relative gene expression data using real-time quantitative PCR and the 2(-Delta Delta C(T)) method. Methods 2001;25:402-8.

[17] Paulino J, Vigia E, Marcelino P, et al. Genetic expression profile of human liver grafts in ischemia-reperfusion injury: comparison of familial amyloidotic polyneuropathy and deceased-donor liver grafts. Transplant Proc 2014;46(6):1678-84.

[18] Walsh KB, Toledo AH, Rivera-Chavez FA, et al. Inflammatory mediators of liver ischemia-reperfusion injury. Exp Clin Transplant 2009; 7(2):78-93.

[19] Klune JR, Tsung A. Molecular biology of liver ischemia/ reperfusion injury: established mechanisms and recent advancements. Surg Clin North Am 2010;90(4):665-77.
[20] Teoh N, Ajamieh H, Wong HJ, et al. Microparticles mediate hepatic ischemia-reperfusion injury and are the targets of Diannexin (ASP8597). Plos One 2014;9(9):e104376.

[21] Yoshimoto K, Tajima H, Ohta T, et al. Increased E-selectin in hepatic ischemia-reperfusion injury mediates liver metastasis of pancreatic cancer. Oncol Rep 2012;28(3):791-6.

[22] Lopez-Castejon G, Brough D. Understanding the mechanism of Il-1 $\beta$ secretion. Cytokine Growth Factor Ver 2011;22: 189-95.

[23] Shroeder K, Tschopp J. The inflammasomes. Cell 2010;140: $821-32$

[24] Szabo G, Csak T. Inflammosomes in Liver disease. J Hepatol 2012;57:642-54.

[25] Bugge JF. Brain death and its implications for management of the potential organ donor. Acta Anaesthesiol Scand 2009;53: 1239-50.

[26] Dziodzio T, Biebl M, Pratschke J. Impact of brain death on ischemia/reperfusion injury in liver transplantation. Curr Opin Organ Transplant 2014;19(2):108-14.

[27] Dinarello CA, Simon A, van der Meer JWM. Treating inflammation by blocking interleukin-1 in a broad Spectrum of diseases. Nat Rev 2012;11:633-52.

[28] Brooks BK, Levy MF, Jennings LW, et al. Influence of donor and recipient gender on the outcome of liver transplantation. Transplantation 1996;62(12):1784-7.

[29] Croome KP, Segal D, Hernandez-Alejandro R, et al. Female donor to male recipient gender discordance results in inferior graft survival: a prospective study of 1,042 liver transplants. J Hepatobiliary Pancreat Sci 2014;21(4):269-74.

[30] Alvaro D, Mancino MG, Onori P, et al. Estrogens and the pathophysiology of the biliary tree. World J Gastroenterol 2006;12(22):3537-45.

[31] Norvell JP, Levitsky MD. Donor and recipient effects on graft and patient survival. Clin Liver Dis 2013;2(4):152-5.

[32] Lai JC, Verna EC, Brown Jr RS, et al. Hepatitis C virusinfected women have a higher risk of advanced fibrosis and graft loss after liver transplantation than men. Hepatology 2011;54(2): 418-24.

[33] Varotti G, Grazi GL, Vetrone G, et al. Causes of early acute graft failure after liver transplantation: analysis of a 17-year singlecentre experience. Clin Transplant 2005;19(4):492-500.

[34] Candinas D, Gunson BK, Nightingale P, et al. Sex mismatch as a risk factor for chronic rejection of liver allografts. Lancet 1995;346(8983):1117-21. 